DO-TH $97 / 26$

November 1997

\title{
Leading log radiative corrections to polarized heavy flavour leptoproduction
}

\author{
I. Schienbein \\ Institut für Physik, Universität Dortmund \\ D-44221 Dortmund, Germany
}

\begin{abstract}
$\underline{\text { Abstract }}$
$\mathcal{O}(\alpha)$ QED radiative corrections to polarized neutral current leptoproduction of heavy quark flavours are calculated in the leading log approximation for HERMES, COMPASS, and HERA kinematics. Besides the inclusive case, we derive general $z_{h}$-differential formulae for the polarized boson gluon fusion process and use them to calculate radiative corrections to semi-inclusive polarized heavy flavour production in the case of HERA kinematics.
\end{abstract}




\section{Introduction}

So far, the polarized gluon density $\Delta g\left(x, Q^{2}\right)$ is nearly unknown, see e.g. [1]. In order to improve this status several experiments are designed to measure polarized heavy flavour production which is directly linked to $\Delta g\left(x, Q^{2}\right)$ already in leading order (LO) QCD via the boson (photon) gluon fusion mechanism. Such measurements will be performed by, e.g., the HERMES Collaboration with its charm detector upgrade [2] and the COMPASS Collaboration at CERN [3]. Using fixed targets these efforts are restricted to rather low energies. A polarized HERA collider mode [4] would allow for measurements in a kinematical region not accessible to any other polarized experiment [5].

Radiative corrections to polarized deep inelastic scattering using HERA and HERMES kinematics have been recently calculated in complete $\mathcal{O}(\alpha)$ and in $\mathcal{O}(\alpha)$ leading log approximation (LLA) [6] disregarding heavy flavour production. However, at small $x \lesssim 10^{-3}$ the heavy (charm) quark contribution $g_{1}^{c}\left(x, Q^{2}\right)$ to the spin structure function $g_{1}^{P}\left(x, Q^{2}\right)$ becomes relevant. Keeping in mind that heavy flavour production processes are semi-inclusive it is recommendable to perform the radiative corrections on a more differential level. As in the unpolarized case [7] we study the momentum $\left(z_{D}=p \cdot p_{D} / p \cdot q\right)$ distributions of the $D$-mesons produced in such events. For this purpose we derive the $z_{h}$-differential expressions $\left(z_{h}=p \cdot p_{h} / p \cdot q\right)$ with general couplings, where $h$ denotes a heavy (charm) quark, for the polarized boson gluon fusion process. This allows us to study electroweak (ew) radiative effects in heavy flavour production. Such effects have been recently investigated in the case of the light flavours $u, d, s$ [6, 8] and there turned out to be sizeable at small Bjorken- $x\left(x=10^{-3}, 10^{-2}\right)$ [6].

Thus, in this paper we calculate radiative corrections to inclusive and semi-inclusive polarized heavy flavour production in LLA for HERMES, COMPASS, and HERA kinematics. Since in leading order QED the polarized and unpolarized splitting functions are the same, i.e. $\Delta P_{e e}(x)=P_{e e}(x)$, the whole formalism can be adopted from the unpolarized case [7] merely replacing the unpolarized Born cross sections by the corresponding 
polarized expressions.

The paper is organized as follows: In Sect. 2 the Born-level expressions for polarized deep inelastic scattering are given and the general $z_{h}$-differential expressions for polarized heavy flavour production via the booson gluon fusion mechanism are derived. In Sect. 3 we provide the theoretical framework to calculate inclusive and semi-inclusive $\mathcal{O}(\alpha)$-QED corrections in LLA, followed by numerical results for the fully massive radiative corrections to heavy flavour production in Sect. 4. Finally, we summarize our results in Sect. 5.

\section{Born cross section}

In our calculations we will restrict on the longitudinal spin asymmetry $d \Delta \sigma=\left(d \sigma\left(\lambda, S_{L}\right)-\right.$ $\left.d \sigma\left(\lambda,-S_{L}\right)\right) / 2$, where $d \sigma\left(\lambda, S_{L}\right)$ denotes the differential Born cross section for deep inelastic scattering of longitudinally polarized leptons off longitudinally polarized nucleons. For definiteness, we consider the process $l\left(k_{1}, \lambda\right) P\left(p, S_{L}\right) \rightarrow l^{\prime}\left(k_{2}\right) X\left(p_{X}\right)$ where $\lambda$ and $k_{1}$ are the helicity and the 4-momentum of the incoming lepton, $S_{L}$ and $p$ the spin and the 4-momentum of the nucleon and $k_{2}$ the 4-momentum of the final lepton. The Born cross section can be written as a contraction of a leptonic tensor $L_{\mu \nu}$ with a hadronic tensor $W^{\mu \nu}$ :

$$
\begin{aligned}
d \sigma^{N C} & =d \sigma^{\gamma \gamma}+2 \operatorname{Re}\left(d \sigma^{\gamma Z}\right)+d \sigma^{Z Z} \\
d \sigma^{C C} & =d \sigma^{W W} \\
d \sigma^{B B^{\prime}} & =\frac{2 \pi \alpha^{2}}{Q^{4}} y \chi_{B} \chi_{B^{\prime}}\left(L_{\mu \nu}^{B B^{\prime}} W_{B B^{\prime}}^{\mu \nu}\right) d x d y
\end{aligned}
$$

with $B, B^{\prime}=\gamma, Z$ or $W, \alpha$ the QED coupling constant, and $x, y, Q^{2}$ the well-known variables in deep inelastic scattering (DIS). $\chi_{B}$ collects some constants and the ratio of $B$-propagator and photon-propagator:

$$
\begin{aligned}
\chi_{\gamma}\left(Q^{2}\right) & =1 \\
\chi_{Z}\left(Q^{2}\right) & =\frac{1}{\left(2 \sin \theta_{W} \cos \theta_{W}\right)^{2}} \frac{Q^{2}}{Q^{2}+M_{Z}^{2}}
\end{aligned}
$$




$$
\chi_{W}\left(Q^{2}\right)=\frac{1}{8\left(\sin \theta_{W}\right)^{2}} \frac{Q^{2}}{Q^{2}+M_{W}^{2}},
$$

where $\theta_{W}$ is the Weinberg angle and $M_{Z}, M_{W}$ are the masses of the $Z^{0}$ and $W$ boson, respectively.

Using a general $\gamma_{\mu}\left(V-A \gamma_{5}\right)$-structure for the leptonic current, $L_{\mu \nu}^{B B^{\prime}}$ reads:

$$
\begin{aligned}
L_{\mu \nu}^{B B^{\prime}}(\lambda) & =\sum_{\lambda^{\prime}} \bar{u}\left(\lambda, k_{1}\right) \Gamma_{\nu}^{B^{\prime}} u\left(\lambda^{\prime}, k_{2}\right) \bar{u}\left(\lambda^{\prime}, k_{2}\right) \Gamma_{\mu}^{B} u\left(\lambda, k_{1}\right) \\
& =2 C_{l}^{B B^{\prime}}\left(k_{1 \mu} k_{2 \nu}+k_{1 \nu} k_{2 \mu}-\left(k_{1} \cdot k_{2}\right) g_{\mu \nu}+2 i \lambda \epsilon_{\mu \nu \rho \sigma} k_{1}^{\rho} k_{2}^{\sigma}\right) .
\end{aligned}
$$

$\Gamma_{\mu}^{B}=\gamma_{\mu}\left(V_{l}^{B}-A_{l}^{B} \gamma_{5}\right)$ and $C_{l}^{B B^{\prime}}=\left(V_{l}^{B}-\lambda p_{l} A_{l}^{B}\right)\left(V_{l}^{B^{\prime}}-\lambda p_{l} A_{l}^{B^{\prime}}\right)$ are built from the vector $(\mathrm{V})$ and axialvector $(\mathrm{A})$ couplings of the fermion-gauge boson vertex $\gamma_{\mu}\left(V_{f}^{B}-A_{f}^{B} \gamma_{5}\right)$. They are given by

\begin{tabular}{l|ll}
$B$ & $V_{f}^{B}$ & $A_{f}^{B}$ \\
\hline$\gamma$ & $Q_{f}$ & 0 \\
$Z$ & $I_{f}^{3}-2 Q_{f} \sin ^{2} \theta_{w}$ & $I_{f}^{3}$ \\
$W$ & 1 & 1
\end{tabular}

where $Q_{f}\left(Q_{e^{-}}=-1, Q_{e^{+}}=+1, Q_{u, c, t}=+2 / 3, Q_{d, s, b}=-1 / 3\right)$ and $I_{f}^{3}\left(I_{e^{+}, u, c, t}^{3}=1 / 2\right.$, $\left.I_{e^{-}, d, s, b}^{3}=-1 / 2\right)$ are the charge and the isospin of the fermion. $p_{l}$ accounts for the fact that the result for $e^{+} p$-scattering can be obtained from the $e^{-} p$-results by the replacement $A_{l}^{B} \rightarrow-A_{l}^{B}$, i.e. $p_{e^{+}}=-1$ and $p_{e^{-}}=1$. Furthermore, it is convenient to define two further combinations of the couplings at this place:

$$
\begin{aligned}
S_{f, \pm}^{B B^{\prime}} & =V_{f}^{B} V_{f}^{B^{\prime}} \pm A_{f}^{B} A_{f}^{B^{\prime}} \\
R_{f, \pm}^{B B^{\prime}} & =\left(V_{f}^{B} A_{f}^{B^{\prime}} \pm V_{f}^{B^{\prime}} A_{f}^{B}\right) / 2
\end{aligned}
$$

such that $C_{l}=S_{l,+}-p_{l} \lambda 2 R_{l,+}$.

Assuming current conservation, the polarized part $W_{\mu \nu}(p, q, S)$ of the hadronic tensor depends in general on five structure functions $g_{i}\left(x, Q^{2}\right)$. In the literature different definitions for the structure functions are used. For a comparison of several conventions see 
[9]. We use the following form:

$$
\begin{aligned}
W_{\mu \nu}^{B B^{\prime}}(p, q, S)= & i \epsilon_{\mu \nu \lambda \sigma} \frac{q^{\lambda} S^{\sigma}}{p \cdot q} g_{1}^{B B^{\prime}}+i \epsilon_{\mu \nu \lambda \sigma} \frac{q^{\lambda}\left(p \cdot q S^{\sigma}-S \cdot q p^{\sigma}\right)}{(p \cdot q)^{2}} g_{2}^{B B^{\prime}} \\
& +\left(g_{\mu \nu}-\frac{q_{\mu} q_{\nu}}{q^{2}}\right) \frac{S \cdot q}{p \cdot q} g_{3}^{B B^{\prime}}-\left(\frac{S \cdot q}{2(p \cdot q)^{2}} p_{\mu}^{\perp} p_{\nu}^{\perp}+\frac{p_{\mu}^{\perp} S_{\nu}^{\perp}+p_{\nu}^{\perp} S_{\mu}^{\perp}}{4 p \cdot q}\right) g_{4}^{B B^{\prime}} \\
& +\left(\frac{p_{\mu}^{\perp} S_{\nu}^{\perp}+p_{\nu}^{\perp} S_{\mu}^{\perp}}{4 p \cdot q}-\frac{S \cdot q}{2(p \cdot q)^{2}} p_{\mu}^{\perp} p_{\nu}^{\perp}\right) g_{5}^{B B^{\prime}}
\end{aligned}
$$

with

$$
p_{\mu}^{\perp}=p_{\mu}-\frac{p \cdot q}{q^{2}} q_{\mu}, \quad S_{\mu}^{\perp}=S_{\mu}-\frac{S \cdot q}{q^{2}} q_{\mu} .
$$

For longitudinally polarized nucleons the nucleon spin vector can be chosen in the nucleon rest frame to be $S_{L}=(0,0,0, M)$. Neglecting terms of the order $\mathcal{O}\left(M^{2} / S\right)$ (S=CMSenergy) one can replace $S_{L}$ by $-\lambda_{N} p$ where $\lambda_{N}$ is the degree of the nucleon polarization 10] and discard $g_{2}$ and $g_{5}$. This yields

$$
W_{\mu \nu}=\lambda_{N}\left(-i \epsilon_{\mu \nu \lambda \sigma} \frac{q^{\lambda} p^{\sigma}}{p \cdot q} g_{1}-\left(g_{\mu \nu}-\frac{q_{\mu} q_{\nu}}{q^{2}}\right) g_{3}+\frac{p_{\mu}^{\perp} p_{\nu}^{\perp}}{p \cdot q} g_{4}\right)
$$

in agreement with the hadronic Tensor given in [1]. Using eq. (11), (31) and (6) one finds the following result for the longitudinal spin asymmetry (polarized part of the Born cross section):

$$
\begin{gathered}
\frac{d^{2} \Delta \sigma}{d x d y}\left(l(\lambda) P\left(\lambda_{N}\right) \rightarrow l^{\prime} X\right)=\frac{4 \pi \alpha^{2}}{Q^{2} x y} \lambda_{N}[ \\
{\left[(2-y) x G_{1}\left(x, Q^{2}\right)+y^{2} x G_{3}\left(x, Q^{2}\right)\right.} \\
\left.+(1-y) G_{4}\left(x, Q^{2}\right)\right]
\end{gathered}
$$

with the structure functions

$$
\begin{aligned}
G_{1}\left(x, Q^{2}\right) & =\lambda \sum_{B, B^{\prime}} C_{l}^{B B^{\prime}} \chi_{B}\left(Q^{2}\right) \chi_{B^{\prime}}\left(Q^{2}\right) g_{1}^{B B^{\prime}}\left(x, Q^{2}\right) \\
G_{3,4}\left(x, Q^{2}\right) & =\sum_{B, B^{\prime}} C_{l}^{B B^{\prime}} \chi_{B}\left(Q^{2}\right) \chi_{B^{\prime}}\left(Q^{2}\right) g_{3,4}^{B B^{\prime}}\left(x, Q^{2}\right) .
\end{aligned}
$$

The structure functions $g_{i}^{B B^{\prime}}$ can be calculated within the quark parton model. The two relevant partonic processes are in leading order (LO) the polarized heavy flavour production via the boson-gluon fusion subprocess $B^{*}(q) g(k) \rightarrow \bar{Q}_{1}\left(p_{1}\right) Q_{2}\left(p_{2}\right)$ and the electron-quark scattering $B^{*} q \rightarrow q$. 


\subsection{Polarized heavy flavour production}

The structure functions $g_{i=1,3,4}^{h}$ for a pair of heavy quarks $Q_{1,2}$ with masses $m_{1,2}$ can be obtained by a convolution of the polarized gluon density $\Delta g$ with the polarized structure functions $\hat{g}_{i=1,3,4}^{h}$ of the partonic subprocess:

$$
\begin{gathered}
g_{1,3}^{h, B B^{\prime}}\left(x, Q^{2}, \mu^{2}\right)=\int_{a x}^{1} \frac{d z_{2}}{z_{2}} \Delta g\left(z_{2}, \mu^{2}\right) \hat{g}_{1,3}^{h, B B^{\prime}}\left(x / z_{2}, Q^{2}, \mu^{2}\right), \\
g_{4}^{h, B B^{\prime}}\left(x, Q^{2}, \mu^{2}\right)=\int_{a x}^{1} \frac{d z_{2}}{z_{2}} z_{2} \Delta g\left(z_{2}, \mu^{2}\right) \hat{g}_{4}^{h, B B^{\prime}}\left(x / z_{2}, Q^{2}, \mu^{2}\right),
\end{gathered}
$$

with $a=1+\left(m_{1}+m_{2}\right)^{2} / Q^{2}$. As usual we identify the scales $\mu_{R}=\mu_{F}=: \mu$ in $\Delta g\left(z_{2}, \mu_{F}\right)$ and $\alpha_{s}\left(\mu_{R}\right)$ (see below).

Following the general framework given in ref. [11], we calculate the polarized partonic structure functions $\hat{g}_{i=1,3,4}^{h, B B^{\prime}}$ in $z_{h}$-differential form $(h=c, b, t)$ by writing the two particle

phase space as $d R_{2}=d z_{h} /(8 \pi)$ with $z_{h}=k \cdot p_{2} / k \cdot q$. The general $z_{h}$-dependence of the polarized structure functions turns out to be the same as in the unpolarized case [12]:

$$
\hat{g}_{i=1,3,4}^{h, B B^{\prime}}\left(x, Q^{2}, \mu^{2} ; z_{h}\right)=\frac{\alpha_{s}\left(\mu^{2}\right)}{4 \pi}\left[\frac{A_{i}^{B B^{\prime}}}{\left(1-z_{h}\right)^{2}}+\frac{B_{i}^{B B^{\prime}}}{z_{h}^{2}}+\frac{C_{i}^{B B^{\prime}}}{\left(1-z_{h}\right)}+\frac{D_{i}^{B B^{\prime}}}{z_{h}}+E_{i}^{B B^{\prime}}\right]
$$

with (suppressing the indices $B B^{\prime}$ )

$$
\begin{aligned}
& A_{1}\left(x, Q^{2}\right)=S_{q,+} \frac{m_{1}^{2}}{Q^{2}} x \\
& C_{1}\left(x, Q^{2}\right)=S_{q,+}\left[\Delta P_{q g}(x)+\frac{\Delta m^{2}}{Q^{2}} x\right] \\
& E_{1}\left(x, Q^{2}\right)=S_{q,+}\left(-2 \Delta P_{q g}(x)\right) \\
& A_{3}\left(x, Q^{2}\right)=-2 \frac{R_{q,+}}{S_{q,+}} A_{1}\left(x, Q^{2}\right) \\
& C_{3}\left(x, Q^{2}\right)=-2 \frac{R_{q,+}}{S_{q,+}} C_{1}\left(x, Q^{2}\right) \\
& E_{3}\left(x, Q^{2}\right)=0 \\
& A_{4}\left(x, Q^{2}\right)=R_{q,+}\left[-4 x^{2} \frac{m_{1}^{2}}{Q^{2}}\left(1+\frac{\Delta m^{2}}{Q^{2}}\right)\right] \\
& C_{4}\left(x, Q^{2}\right)=R_{q,+} 2 x\left[-2 \Delta P_{q g}(x)+\frac{\Delta m^{2}}{Q^{2}}(1-4 x)-2 x \frac{\Delta m^{4}}{Q^{4}}\right]
\end{aligned}
$$




$$
\begin{aligned}
E_{4}\left(x, Q^{2}\right) & =0 \\
B_{i={ }_{3,4}^{1}}\left(x, Q^{2}\right) & = \pm A_{i}\left(x, Q^{2}\right)\left[m_{1} \leftrightarrow m_{2}\right] \\
D_{i={ }_{3,4}^{1}}\left(x, Q^{2}\right) & = \pm C_{i}\left(x, Q^{2}\right)\left[m_{1} \leftrightarrow m_{2}\right]
\end{aligned}
$$

where $\Delta m^{n} \equiv m_{2}^{n}-m_{1}^{n}, m_{1,2}$ being the mass of the heavy quark $Q_{1,2}$ and $\Delta P_{q g}(x)=$ $(2 x-1) / 2$ the polarized $g \rightarrow q$ splitting function.

The $z_{h}$-integrated results in [1] are easily recovered if one notices that $1 /\left(1-z_{h}\right) \rightarrow$ $L=\ln \left(1-z_{h, \min }\right) /\left(1-z_{h, \max }\right), 1 / z_{h} \rightarrow \tilde{L}=\ln z_{h, \max } / z_{h, \min }=L\left[m_{1} \leftrightarrow m_{2}\right], 1 /\left(1-z_{h}\right)^{2} \rightarrow$ $v \bar{v} W^{2} / m_{1}^{2}$ and $1 / z_{h}^{2} \rightarrow v \bar{v} W^{2} / m_{2}^{2}$ with the $z_{h}$-integration boundaries [14]

$$
z_{h_{\min , \max }}\left(x, Q^{2}\right)=\frac{1}{2}\left[1+\Delta m^{2} / W^{2} \pm v \bar{v}\right]
$$

where $v^{2}\left(x, Q^{2}\right)=1-\left(m_{1}+m_{2}\right)^{2} / W^{2}, \bar{v}^{2}\left(x, Q^{2}\right)=1-\left(m_{1}-m_{2}\right)^{2} / W^{2}$ and $W^{2}\left(x, Q^{2}\right)=$ $\frac{Q^{2}}{x}(1-x)$. In the following we are only interested in neutral current charm production, i.e., $m_{1}=m_{2}=m_{c}, \bar{v}=1$ and $\tilde{L}=L=\ln (1+v) /(1-v)$. In this case the $z_{h}$-integrated partonic structure functions read:

$$
\begin{aligned}
& \hat{g}_{1}^{c, B B^{\prime}}\left(x, Q^{2}, \mu^{2}\right)=\frac{\alpha_{s}\left(\mu^{2}\right)}{4 \pi} S_{q,+}^{B B^{\prime}}\left[2 \Delta P_{q g}(x) L+v(3-4 x)\right] \\
& \hat{g}_{3}^{c, B B^{\prime}}\left(x, Q^{2}, \mu^{2}\right)=\hat{g}_{4}^{c, B B^{\prime}}\left(x, Q^{2}, \mu^{2}\right)=0 .
\end{aligned}
$$

\section{$2.2 \quad$ Electron-quark scattering}

For completeness we list the LO neutral current results for electron quark scattering [11]]:

$$
\begin{aligned}
g_{1}^{B B^{\prime}}\left(x, Q^{2}\right) & =\frac{1}{2} \sum_{q=u, d, s} S_{q,+}^{B B^{\prime}}\left[\Delta q\left(x, Q^{2}\right)+\Delta \bar{q}\left(x, Q^{2}\right)\right] \\
g_{3}^{B B^{\prime}}\left(x, Q^{2}\right) & =-\sum_{q=u, d, s} R_{q,+}^{B B^{\prime}}\left[\Delta q\left(x, Q^{2}\right)-\Delta \bar{q}\left(x, Q^{2}\right)\right] \\
g_{4}^{B B^{\prime}}\left(x, Q^{2}\right) & =2 x g_{3}^{B B^{\prime}}\left(x, Q^{2}\right) .
\end{aligned}
$$

\footnotetext{
${ }^{1}$ Note that $R_{q,+}=-R_{q} / 2$ with $R_{q}$ defined in [11].
} 


\section{Radiative corrections in LLA}

As already mentioned, in $\mathcal{O}(\alpha)$-LLA the structure of the QED-corrections to polarized DIS is the same as in the unpolarized case, because $\Delta P_{e e}(x)=P_{e e}(x)=\left[\frac{1+x^{2}}{1-x}\right]_{+}$. We therefore restrict ourselves to give the formulae that we have used in this work. For details we refer the reader to the literature [7, 13].

We are using leptonic variables

$$
q_{l} \equiv p_{e}-p_{e}^{\prime}, \quad Q_{l}^{2} \equiv-q_{l}^{2}, \quad y_{l}=\frac{p \cdot q_{l}}{p \cdot p_{e}}, \quad x_{l} \equiv \frac{Q_{l}^{2}}{2 p \cdot q_{l}}=\frac{Q_{l}^{2}}{S y_{l}},
$$

which are reconstructed by the 4-momentum $p_{e}^{\prime}$ of the observed electron and the 4momenta $p_{e}, p$ of the initial state electron and proton. As usual $S=\left(p+p_{e}\right)^{2}$ is the center-of-mass (CMS) energy.

To describe semi-inclusive processes we further define the Lorentz-invariant variable

$$
z_{D} \equiv z_{D, l}=\frac{p \cdot p_{D}}{p \cdot q_{l}}
$$

which is built from the 4 -momentum $p_{D}$ of the produced $D$-meson. In the rest frame of the proton $z_{D}$ is the energy fraction carried by the $D$-meson with respect to the energy of the boson.

The rescaled variables for the partonic subprocess like ,e.g.,

$$
\begin{aligned}
e\left(\hat{p}_{e}=z_{1} p_{e}\right) & +g\left(\hat{p}=z_{2} p\right) \longrightarrow e\left(\hat{p}_{e}^{\prime}=p_{e}^{\prime} / z_{3}\right)+c\left(p_{c}=P_{D} / z_{4}\right)+X \text { read: } \\
\hat{q} & =\hat{p}_{e}-\hat{p}_{e}^{\prime}, \quad \hat{Q}^{2}=-\hat{q}^{2}=\frac{z_{1}}{z_{3}} Q_{l}^{2}, \quad \hat{y}=\frac{\hat{p} \hat{q}}{\hat{p} \hat{p}_{e}}=\frac{z_{1} z_{3}-1+y_{l}}{z_{1} z_{3}}, \\
\hat{x} & =\frac{\hat{Q}^{2}}{2 \hat{p} \cdot \hat{q}}=\frac{\hat{Q}^{2}}{\hat{S} \hat{y}}=\frac{z_{1} x_{l} y_{l}}{z_{2}\left(z_{1} z_{3}-1+y_{l}\right)}, \quad \hat{S}=\left(\hat{p}_{e}+\hat{p}\right)^{2}=z_{1} z_{2} S, \\
\hat{z}_{c} & =\hat{p} \cdot p_{c} / \hat{p} \cdot \hat{q}=\frac{z_{D} y_{l} z_{3}}{z_{4}\left(z_{1} z_{3}+y_{l}-1\right)} \equiv \frac{z_{D}}{z_{4}} r
\end{aligned}
$$

with $r$ defined by

$$
r \equiv \frac{y_{l} z_{3}}{z_{1} z_{3}+y_{l}-1} .
$$

The index $l$ to denote leptonic variables will be suppressed from now on. 


\subsection{Inclusive case}

The corrections due to initial state radiation (ISR) and final state radiation (FSR) can be written as [7]

$$
\begin{aligned}
\frac{d^{2} \Delta \sigma^{i}}{d x d y}= & \frac{\alpha L_{e}}{2 \pi} \int_{z_{1}^{\text {min }}}^{1} d z_{1}\left[\frac{1+z_{1}^{2}}{1-z_{1}}\left(\Delta \sigma_{0}\left(z_{1}, 1\right)-\Delta \sigma_{0}(1,1)\right)\right] \\
& +\frac{\alpha L_{e}}{2 \pi} H\left(z_{1}^{\min }\right) \Delta \sigma_{0}(1,1), \\
\frac{d^{2} \Delta \sigma^{f}}{d x d y}= & \frac{\alpha L_{e}}{2 \pi} \int_{z_{3}^{\min }}^{1} d z_{3}\left[\frac{1+z_{3}^{2}}{1-z_{3}}\left(\Delta \sigma_{0}\left(1, z_{3}\right)-\Delta \sigma_{0}(1,1)\right)\right] \\
& +\frac{\alpha L_{e}}{2 \pi} H\left(z_{3}^{\text {min }}\right) \Delta \sigma_{0}(1,1),
\end{aligned}
$$

with $\alpha$ the electromagnetic coupling, $L_{e}=\ln Q^{2} / m_{e}^{2}\left(m_{e}=\right.$ electron mass) and

$$
H(z)=-\int_{0}^{z} d x \frac{1+x^{2}}{1-x}=2 \ln (1-z)+z+\frac{1}{2} z^{2} .
$$

$\Delta \sigma_{0}$ depends on the hard scattering process.

1. Boson gluon fusion (heavy flavour production):

$$
\Delta \sigma_{0}\left(z_{1}, z_{3}\right)=\int_{z_{2}^{\min }}^{1} d z_{2} \Delta g\left(z_{2}, \mu^{2}\right) \frac{\partial(\hat{x}, \hat{y})}{\partial(x, y)} \frac{d^{2} \Delta \hat{\sigma}}{d \hat{x} d \hat{y}},
$$

with the partonic cross section

$$
\frac{d^{2} \Delta \hat{\sigma}}{d \hat{x} d \hat{y}}=\frac{4 \pi \alpha^{2}}{\hat{S} \hat{x}^{2} \hat{y}^{2}} \lambda_{N}\left[\hat{y}(2-\hat{y}) \hat{x} \hat{G}_{1}\left(\hat{x}, \hat{Q}^{2}\right)\right]
$$

where

$$
\hat{G}_{1}\left(\hat{x}, \hat{Q}^{2}\right)=\lambda \sum_{B, B^{\prime}} C_{l}^{B B^{\prime}} \chi_{B}\left(\hat{Q}^{2}\right) \chi_{B^{\prime}}\left(\hat{Q}^{2}\right) \hat{g}_{1}^{B B^{\prime}}\left(\hat{x}, Q^{2}\right)
$$

$\left(\left(\hat{g}_{1}\left(\hat{x}, \hat{Q}^{2}\right)\right.\right.$ from eq. (12) $)$ and the Jacobian

$$
\frac{\partial(\hat{x}, \hat{y})}{\partial(x, y)}=\frac{y}{z_{2} z_{3}\left(z_{1} z_{3}-1+y\right)} .
$$


The integration bounds are given by:

$$
\begin{aligned}
z_{1}^{\text {min }} & =\frac{1-y}{1-x y}+\frac{4 m_{c}^{2}}{S(1-x y)} \\
z_{3}^{\text {min }} & =\frac{1-y+x y}{1-4 m_{c}^{2} / S} \\
z_{2}^{\text {min }}\left(z_{1}, z_{3}\right) & =\left(1+\frac{4 m_{c}^{2}}{Q^{2}} \frac{z_{3}}{z_{1}}\right) z_{1} x y \frac{1}{z_{1} z_{3}+y-1}
\end{aligned}
$$

\section{Light flavour excitation:}

In the case of the scattering off the light (massless) flavours $(u, d, s)$ the partonic cross section is proportional to $\delta(1-\hat{x}$ ) and one easily finds (of course $\Delta g \rightarrow \Delta q, q=u, d, s$ ) $\Delta \sigma_{0}\left(z_{1}, z_{3}\right)=\frac{4 \pi \alpha^{2}}{S x^{2} y^{2}} \frac{y}{\hat{y} z_{1}^{2}} \lambda_{N}\left(\hat{y}(2-\hat{y}) \bar{z}_{2} G_{1}\left(\bar{z}_{2}, \hat{Q}^{2}\right)+\hat{y}^{2} \bar{z}_{2} G_{3}\left(\bar{z}_{2}, \hat{Q}^{2}\right)+(1-\hat{y}) G_{4}\left(\bar{z}_{2}, \hat{Q}^{2}\right)\right)$

with $\bar{z}_{2}=\frac{z_{1} x y}{z_{1} z_{3}+y-1}$ and the structure functions $G_{i}$ from eq. (8) and (13). The integration bounds can be obtained from the above $z_{1,3}^{\min }$ by setting $m_{c} \rightarrow 0$.

\section{$3.2 z_{D}$-differential case}

In this subsection we consider $z_{D}$-differential polarized heavy flavour production. The cross sections for ISR and FSR have the same structure as in eq. (18) and (19) with a $z_{D}$-dependent function $\Delta \sigma_{0}[7]$ :

$$
\begin{aligned}
\frac{d^{3} \Delta \sigma^{i}}{d x d y d z_{D}}= & \frac{\alpha L_{e}}{2 \pi} \int_{z_{1}^{\text {min }}}^{1} d z_{1}\left[\frac{1+z_{1}^{2}}{1-z_{1}}\left(\Delta \sigma_{0}\left(z_{1}, 1 ; z_{D}\right)-\Delta \sigma_{0}\left(1,1 ; z_{D}\right)\right)\right] \\
& +\frac{\alpha L_{e}}{2 \pi} H\left(z_{1}^{\text {min }}\right) \Delta \sigma_{0}\left(1,1 ; z_{D}\right) \\
\frac{d^{3} \Delta \sigma^{f}}{d x d y d z_{D}}= & \frac{\alpha L_{e}}{2 \pi} \int_{z_{3}^{\text {min }}}^{1} d z_{3}\left[\frac{1+z_{3}^{2}}{1-z_{3}}\left(\Delta \sigma_{0}\left(1, z_{3} ; z_{D}\right)-\Delta \sigma_{0}\left(1,1 ; z_{D}\right)\right)\right] \\
& +\frac{\alpha L_{e}}{2 \pi} H\left(z_{3}^{\text {min }}\right) \Delta \sigma_{0}\left(1,1 ; z_{D}\right)
\end{aligned}
$$

with

$$
\Delta \sigma_{0}\left(z_{1}, z_{3} ; z_{D}\right)=\int_{z_{2}^{\min }}^{1} d z_{2} \Delta g\left(z_{2}, \mu^{2}\right) \int_{z_{4}^{\min }}^{z_{4}^{\max }} d z_{4} \frac{\partial\left(\hat{x}, \hat{y}, \hat{z_{c}}\right)}{\partial\left(x, y, z_{D}\right)} \frac{d^{3} \Delta \hat{\sigma}}{d \hat{x} d \hat{y} d \hat{z}_{c}} D_{c}\left(z_{4}\right)
$$


The partonic cross section is given by

$$
\begin{aligned}
& \frac{d^{3} \Delta \hat{\sigma}}{d \hat{x} d \hat{y} d \hat{z_{c}}}=\frac{4 \pi \alpha^{2}}{\hat{S} \hat{x}^{2} \hat{y}^{2}} \lambda_{N}\left[\hat{y}(2-\hat{y}) \hat{x} \hat{G}_{1}\left(\hat{x}, \hat{Q}^{2}, \hat{z}_{c}\right)+\hat{y}^{2} \hat{x} \hat{G}_{3}\left(\hat{x}, \hat{Q}^{2}, \hat{z}_{c}\right)\right. \\
& \left.+(1-\hat{y}) \hat{G}_{4}\left(\hat{x}, \hat{Q}^{2}, \hat{z}_{c}\right)\right],
\end{aligned}
$$

inserting the $z_{h}$-differential structure functions from eq. (10) in

$$
\begin{aligned}
\hat{G}_{1}\left(\hat{x}, \hat{Q}^{2}, \hat{z}_{c}\right) & =\lambda \sum_{B, B^{\prime}} C_{l}^{B B^{\prime}} \chi_{B}\left(\hat{Q}^{2}\right) \chi_{B^{\prime}}\left(\hat{Q}^{2}\right) \hat{g}_{1}^{B B^{\prime}}\left(\hat{x}, Q^{2}, \hat{z}_{c}\right) \\
\hat{G}_{3,4}\left(\hat{x}, \hat{Q}^{2}, \hat{z}_{c}\right) & =\sum_{B, B^{\prime}} C_{l}^{B B^{\prime}} \chi_{B}\left(\hat{Q}^{2}\right) \chi_{B^{\prime}}\left(\hat{Q}^{2}\right) g_{3,4}^{B B^{\prime}}\left(\hat{x}, Q^{2}, \hat{z}_{c}\right) .
\end{aligned}
$$

The Jacobian in eq. (28) can easily be calculated as

$$
\frac{\partial\left(\hat{x}, \hat{y}, \hat{z}_{c}\right)}{\partial\left(x, y, z_{D}\right)}=\frac{\partial(\hat{x}, \hat{y})}{\partial(x, y)} \frac{\partial \hat{z}_{c}}{\partial z_{D}}=\frac{y^{2}}{z_{2} z_{4}\left(z_{1} z_{3}-1+y\right)^{2}} .
$$

The additional integration bounds read:

$$
\begin{aligned}
& z_{4}^{\max }=\min \left[1, \frac{z_{D} r}{\hat{z}_{c}^{\min }}\right], \\
& z_{4}^{\min }=\min \left[z_{4}^{\max }, \frac{z_{D} r}{\hat{z}_{c}^{\max }}\right]
\end{aligned}
$$

with $r$ from eq. (17) and $\hat{z}_{c}^{\min , \max }=z_{h=c}^{\min , \max }\left(\hat{x}, \hat{Q}^{2}\right)$ from eq. (11).

For $D_{c}(z)$, we use a Peterson et al. fragmentation function [15]

$$
D_{c}(z)=N\left\{z\left[1-z^{-1}-\varepsilon_{c} /(1-z)\right]^{2}\right\}^{-1}
$$

normalized to $\int_{0}^{1} d z D_{c}(z)=1$, i. e. ,

$$
N^{-1}=\frac{\left(\varepsilon_{c}^{2}-6 \varepsilon_{c}+4\right)}{\left(4-\varepsilon_{c}\right) \sqrt{4 \varepsilon_{c}-\varepsilon_{c}^{2}}}\left\{\arctan \frac{\varepsilon_{c}}{\sqrt{4 \varepsilon_{c}-\varepsilon_{c}^{2}}}+\arctan \frac{2-\varepsilon_{c}}{\sqrt{4 \varepsilon_{c}-\varepsilon_{c}^{2}}}\right\}+\frac{1}{2} \ln \varepsilon_{c}+\frac{1}{4-\varepsilon_{c}} .
$$

\section{Numerical results}

The production of heavy flavours will be measured in several future experiments, e.g., the charm upgrade of the HERMES experiment [2], the COMPASS experiment [3] and 
a possible polarized HERA collider mode [ [N]. In the following, we discuss the radiative corrections to polarized heavy flavour leptoproduction in the kinematical regions of these experiments.

As usual we introduce the correction factor $\delta$ by $d \Delta \sigma=d \Delta \sigma^{0}(1+\delta)$, i. e., $\delta=$ $\delta^{i}+\delta^{f}+\ldots$ with

$$
\delta^{i, f}(x, y)=\frac{d^{2} \Delta \sigma^{i, f}}{d x d y} / \frac{d^{2} \Delta \sigma^{0}}{d x d y}
$$

or, in the $z_{D}$-differential case,

$$
\delta^{i, f}\left(x, y, z_{D}\right)=\frac{d^{3} \Delta \sigma^{i, f}}{d x d y d z_{D}} / \frac{d^{3} \Delta \sigma^{0}}{d x d y d z_{D}} .
$$

The labels "i" and "f" stand for "initial" and "final" state radiation, $d \Delta \sigma^{0}$ denotes the Born cross section under consideration.

In the following figures we employ the polarized parton densities GRSV(LO) 'Standard' [16] with a positive definite polarized gluon and GS(LO) 'C' [17] with a non-positive definite gluon. It should be stressed again that both of these different parametrizations describe present data. Furthermore, theoretical uncertainties due to different choices of the charm mass $m_{c}$ and the factorization scale $\mu$ can be sizeable not only in the cross sections but also in the ratio $\delta$. In all figures the charm mass has been set to $m_{c}=1.5$ $\mathrm{GeV}$. An appropriate choice of $\mu$ is not known. In order to find a factorization scale $\mu$ leading to a good perturbative stability affords the knowledge of the next-to-leading order corrections to polarized heavy flavour production which have not yet been calculated. Thus, we use the two factorization scales $\mu_{1}^{2}=4 m_{c}^{2}$ and $\mu_{2}^{2}=Q^{2}+4 m_{c}^{2}$, which have been favoured in the unpolarized case 18. For completeness, all figures have been calculated with $\lambda=\lambda_{N}=1$.

\subsection{HERMESC}

The detector upgrade of the HERMES experiment [2] is dedicated to measure polarized leptoproduction of open charm via the scattering of longitudinally polarized elec- 
trons $\left(E_{e}=27.5 \mathrm{GeV}\right)$ off a longitudinally polarized fixed nucleon target, i. e. , at $\sqrt{S}=7.4 \mathrm{GeV}$. In fig. 目 $\left[m_{c}=1.5 \mathrm{GeV}, \mu^{2}=Q^{2}+4 m_{c}^{2} \approx 4 m_{c}^{2}\right.$, parton distributions: GRSV(LO) 'Standard' (solid line), GS(LO) 'C' (dashed line)] we show the radiative corrections in $\mathcal{O}(\alpha)$-LLA to this process according to eq. (18)-(20) for two values of Bjorken- $x$ in dependence of $y$. From the threshold condition $\hat{s}=\left(p_{c}+p_{\bar{c}}\right)^{2} \geq 4 m_{c}^{2}$ follows $y \geq 4 m_{c}^{2} /(S(1-x))=: y_{\min } \approx 0.16 /(1-x)$. In the range $y_{\min } \leq y \leq 0.4$ the corrections are big and strongly depend on the parametrization of the polarized gluon. In the case of the GS parton distributions the correction factor $\delta$ diverges at $y \approx 0.30$ for $x=10^{-2}$ and $y \approx 0.35$ for $x=10^{-1}$ due to a change of sign of the Born cross section in the denominator. However, in this range the cross section $d \Delta \sigma$ is small. In the limit $y \rightarrow y_{\min } d \Delta \sigma$ vanishes. In the physically relevant region $y \gtrsim 0.5$ the corrections are smaller than $20 \%$ further decreasing with increasing $y$. Uncertainties due to the parton distributions are smaller than $5 \%$. Using a factorization scale $\mu^{2}=\hat{s}$ leads to negligible changes in figure 1 .

\subsection{COMPASS}

The COMPASS experiment [3] is going to measure polarized open charm muoproduction at an energy of $\sqrt{S}=14.1 \mathrm{GeV}\left(E_{\mu}=100 \mathrm{GeV}\right)$. The radiative corrections for muoproduction can be obtained by simply replacing $L_{e}=\ln Q^{2} / m_{e}^{2} \rightarrow \ln Q^{2} / m_{\mu}^{2}=L_{\mu}$. Since nothing else is changed the corrections $\delta(x, y)$ are expected to be similar to fig. 1 but suppressed by the factor $L_{\mu} / L_{e}<0.5$. Fig. $2\left[m_{c}=1.5 \mathrm{GeV}, \mu^{2}=Q^{2}+4 m_{c}^{2} \approx 4 m_{c}^{2}\right.$, parton distribution: GRSV(LO) 'Standard'] shows the correction factor $\delta\left(Q^{2}, \nu\right)\left(y=\nu / E_{\mu}\right.$, $\left.x=Q^{2} / 2 M \nu\right)$ for the $\left(\nu, Q^{2}\right)$-range given in table 3.1 of [3]. Only for $\nu=40 \mathrm{GeV}$ and $Q^{2} \geq 6 \mathrm{GeV}^{2}$ the corrections exceed $5 \%$. 


\subsection{Polarized HERA collider mode}

In a polarized HERA collider mode longitudinally polarized electrons are scattered off longitudinally polarized protons with a center-of-mass energy $\sqrt{S}=300 \mathrm{GeV}$. One of the main goals of a polarized HERA is the measurement of the spin dependent structure function $g_{1}\left(x, Q^{2}\right)$ at values of $\left(x, Q^{2}\right)$ which are not accessible to other experiments [5]. In the range of small $x \lesssim 10^{-3}$ the charm contribution $g_{1}^{c}\left(x, Q^{2}\right)$ to $g_{1}^{P}\left(x, Q^{2}\right)$ has to be accounted for.

In fig. [3 the charm contribution to the radiative corrections to $d^{2} \Delta \sigma / d x d y(e p \rightarrow e X)$ is illustrated. On the right side we show the electromagnetic (em) cross sections $d \Delta \sigma^{0, i}(e p \rightarrow$ $e X$ ), including the $u, d, s$ contributions from electron quark scattering (eq. (28)) and the charm contribution due to the photon gluon fusion (eq. (20)), at $\sqrt{S}=300 \mathrm{GeV}$ for $x=10^{-2}, 10^{-3}$, and $10^{-4}$, using the GRSV(LO) 'S' [16 (solid line) and the GS(LO) 'C' [17] (dashed line) parton densities and a factorization scale $\mu^{2}=Q^{2}+4 m_{c}^{2}$ with $m_{c}=1.5$ $\mathrm{GeV}$ (for the charm contribution). The cross sections for FSR have similar shapes as for ISR. The corresponding correction factors $\delta(x, y)$ can be seen on the left hand side. The dotted lines have been calculated without heavy (charm) quark contribution. Employing the GRSV parton distributions they agree with the dashed lines in the figures 11 and 12 in [6]. However, for $x \lesssim 10^{-2}$ the corrections strongly depend on the choice of the parametrization of the polarized parton densities as can be seen by comparison of the solid with the dashed lines. This point has not been hinted at in the literature. The charm contribution to the radiative corrections is roughly $10 \%$ in regions where the corrections do not exceed $50 \%$ and even bigger otherwise.

Similar to the unpolarized case $\left(F_{2}^{c}\right)[19,20$ it would be interesting, albeit very difficult, to measure $g_{1}^{c}$ in order to constrain the polarized gluon density $\Delta g\left(x, Q^{2}\right)$ in the proton at small $x$. Fig. 1 shows the (inclusive) radiative corrections to polarized charm production via the photon gluon fusion. $Z$-exchange has been neglected, since $\hat{g}_{3}^{c}=\hat{g}_{4}^{c}=0$ (inclusive

case, see eq. (12)) and $\hat{g}_{1}^{c, \gamma \gamma} \approx \hat{g}_{1}^{c}$. In contrast to unpolarized heavy flavour production 
[7] the theoretical uncertainties are not under control. The use of the two factorization scales $\mu_{1}^{2}=4 m_{c}^{2}$ and $\mu_{2}^{2}=Q^{2}+4 m_{c}^{2}$ 18 and the parton distributions GRSV(LO) 'S' and GS(LO) 'C' in each case results in rather different corrections, especially for $x=10^{-3}$. This behaviour is not only due to the unknown NLO corrections but mainly due to the almost entirely unconstrained $\Delta g\left(x, Q^{2}\right)$.

As has been already stressed in [7] measurements of heavy quark production processes (one hadron inclusive processes) are of course differential in the momentum of the (observed) heavy quark, recommending to perform the radiative corrections on the same differential level. Thus, we have repeated the semi-inclusive $z_{D}$-differential analysis of our study of unpolarized heavy quark production. Contrary to the inclusive case, $g_{3}$ and $g_{4}$ do not vanish (see eq. (10)) and should not be neglected. Allthough $g_{4}\left(x, Q^{2}\right) \approx$ $\chi_{Z}\left(Q^{2}\right) g_{4}^{\gamma Z}\left(x, Q^{2}\right)$ is suppressed at small $Q^{2} \ll M_{Z}^{2}$ by the factor $\chi_{Z}\left(Q^{2}\right) \approx 0.7 \frac{Q^{2}}{Q^{2}+M_{Z}^{2}}$ its weight relativ to $g_{1}\left(x, Q^{2}\right)$ in the cross section is (up to terms of the order $\mathcal{O}\left(y^{2}\right)$ )

$$
\frac{(1-y) g_{4}\left(x, Q^{2}\right)}{2 x y g 1\left(x, Q^{2}\right)} \approx \frac{\chi_{Z}}{2 x y} \frac{g_{4}^{\gamma Z}}{g 1}
$$

due to the structure of the hadronic tensor. The same holds for the radiative corrections where the corresponding factor in $\Delta \sigma_{0}\left(z_{1}, z_{3}\right)$ reads $\frac{(1-\hat{y}) g_{4}\left(\bar{z}_{2}, \hat{Q}^{2}\right)}{2 x y g_{1}\left(\bar{z}_{2}, \hat{Q}^{2}\right)}$. The factor $\chi_{Z} / 2 x y \approx 0.7 S x y / 2 x y \cdot 1 /\left(Q^{2}+M_{Z}^{2}\right)$ is varying between approximately $0.7\left(Q^{2}=S=10^{5}\right.$ $\left.\mathrm{GeV}^{2}\right)$ and $7\left(Q^{2} \ll M_{Z}^{2}(\right.$ small $\left.x)\right)$. For this reason it is (a priori) not allowed to disregard $g_{4}$ (,i.e. , contributions from $Z$-exchange)2]. However, in the case of $z_{D}$-differential heavy flavour production the differences between em and ew radiative corrections turn out to be negligible. This could be expected because the electroweak effects from $g_{3,4}$ have to vanish after $z_{D}$-integration (see eq. (12). In fig. 5 we show the $z_{D}$-differential radiative corrections according to eq. (26)-(28) after additional integration over the kinematical range $10 \mathrm{GeV}^{2} \leq Q^{2} \leq 100 \mathrm{GeV}^{2}, 0.01 \leq y \leq 0.7$. The left hand side shows the correction factor $\delta\left(z_{D}\right)$ (initial and final state radiation), employing the GRSV(LO) 'S' [16] (solid line) and the GS(LO) 'C' 17 (dashed line) parton distributions. Again we have chosen

\footnotetext{
${ }^{2}$ The importance of the electroweak contributions can be seen in fig. 12 of [6]. We can confirm this result and are grateful to D. Bardin for discussions on this point.
} 
$\mu^{2}=Q^{2}+4 m_{c}^{2}$ with $m_{c}=1.5 \mathrm{GeV}$. For $D_{c}$ a Peterson et al. fragmentation function 15] with $\varepsilon=0.15$ has been taken. On the right side of fig. 5 the $z_{D^{-}}$-dependence of the cross sections $d \Delta \sigma^{0, i}$ is displayed. As in the inclusive case the ISR and FSR cross sections have similar shapes. Apart from irrelevant regions of the phase space where the Born cross section vanishes the corrections amount between $20 \%$ and $-40 \%$. Applying suitable (depending on the parton distribution) cuts, e. g. , $0.2 \leq z_{D} \leq 0.8$ it is possible to further reduce the corrections as can be seen from the rhs of fig. 5 .

To conclude, at small $x$ it is (up to now) only possible to perform calculations of radiative corrections to charm production modulo the choice of the parametrization of the polarized gluon. The situation will improve as soon as the gluon distribution becomes more constrained (for $x \lesssim 10^{-2}$ ) and the NLO expressions for polarized heavy flavour production are calculated.

\section{Summary}

We have calculated fully massive QED corrections to polarized heavy flavour leptoproduction in $\mathcal{O}(\alpha)$-LLA for HERMES, COMPASS, and HERA kinematics. The radiative corrections to the first two experiments are smaller than $20 \%$ and $5 \%$ respectively in relevant realms of the phase space and the theoretical uncertainties due to different choices of the parton distributions and the factorization scale are under control. For HERA kinematics the situation changes if $x<10^{-2}$. In this case all results strongly depend on the chosen set of parton distributions. However, for any fixed parton distribution we find the charm contribution to the corrections to a measurement of $g_{1}^{P}\left(x, Q^{2}\right)$ non-negligible (for $\left.x \lesssim 10^{-3}\right)$. Finally, we have discussed radiative corrections to $g_{1}^{c}\left(x, Q^{2}\right)$ at a polarized HERA in analogy to $F_{2}^{c}\left(x, Q^{2}\right)$ [19, 20] in the unpolarized case. Besides the inclusive case, we have derived general $z_{h}$-differential expressions for the polarized boson gluon fusion process. These formulae have been used to study $z_{D}$-differential corrections, keeping in mind that heavy flavour production is a semi-inclusive process recommending to calculate 
the corrections on the same differential level. These corrections turned out to lie between $20 \%$ and $-40 \%$ apart from regions of the phase space where the Born cross section is small.

\section{Acknowledgements}

It is a pleasure to thank E. Reya and M. Glück for advice and useful discussions. This work has been supported by the Graduiertenkolleg 'Erzeugung und Zerfälle von Elementarteilchen' of the University of Dortmund. 


\section{References}

[1] T. Gehrmann, Summary of the Working Group IV "Spin Physics" of the International Workshop on "Deep Inelastic Scattering and QCD" (DIS '97), hep-ph/9706351; M. Stratmann, in Proceedings of the 2nd Topical Workshop on 'DIS off Polarized Targets: Theory Meets Experiment', Zeuthen, Germany, 1-5 Sep 1997 (hep-ph/9710379).

[2] The HERMES Collab., The HERMES Charm Upgrade Program, HERMES 97-004.

[3] G. Baum et al., COMPASS Collab., CERN/SPSLC 96-14.

[4] J. Feltesse and A. Schäfer, in Proceedings of the workshop on Future Physics at HERA, DESY, 1996, G. Ingelman, A. De Roeck and R. Klanner (ed.) vol. 2, p. 760.

[5] R. Ball et al., in Proceedings of the workshop on Future Physics at HERA, DESY, 1996, G. Ingelman, A. De Roeck and R. Klanner (ed.) vol. 2, p. 777.

[6] D. Bardin, J. Blümlein, P. Christova, and L. Kalinovskaya, Nucl. Phys. B506 (1997) 295.

[7] I. Schienbein, DO-TH 97/05, hep-ph/9705322, Eur. Phys. J. C(to appear), DOI $10.1007 / \mathrm{s} 100529800714$.

[8] I. Akushevich, A. Ilyichev, and N. Shumeiko, hep-ph/9710486.

[9] J. Blümlein and N. Kochelev, Phys. Lett. B381 (1996) 296; Nucl. Phys. B498 (1997) 285.

[10] A. V. Manohar, An Introduction to spin dependent deep inelastic scattering. Lectures given at Lake Louise Winter Inst., Lake Louise, Canada, Feb 23-29, 1992, hep-ph/9204208.

[11] W. Vogelsang and A. Weber, Nucl. Phys. B362 (1991) 3.

[12] S. Kretzer and I. Schienbein, Phys. Rev. D56 (1997) 1804. 
[13] See, for example: J. Blümlein, Z. Phys. C47 (1990) 89;

J. Kripfganz, H.-J. Möhring, and H. Spiesberger, Z. Phys. C49 (1991) 501.

[14] G. Schuler, Nucl. Phys. B299 (1988) 21.

[15] C. Peterson et al., Phys. Rev. D27 (1983) 105.

[16] M. Glück, E. Reya, M. Stratmann, and W. Vogelsang, Phys. Rev. D53 (1996) 4775.

[17] T. Gehrmann and W. J. Stirling, Phys. Rev. D53 (1996) 6100.

[18] M. Glück, E. Reya, and M. Stratmann, Nucl. Phys. B422 (1994) 37;

A. Vogt, DESY 96-012, hep-ph/9601352.

[19] C. Adloff et al., H1 Collab., Z. Phys. C72 (1996) 593.

[20] J. Breitweg et al., ZEUS Collab., Phys. Lett. B407 (1997) 402. 


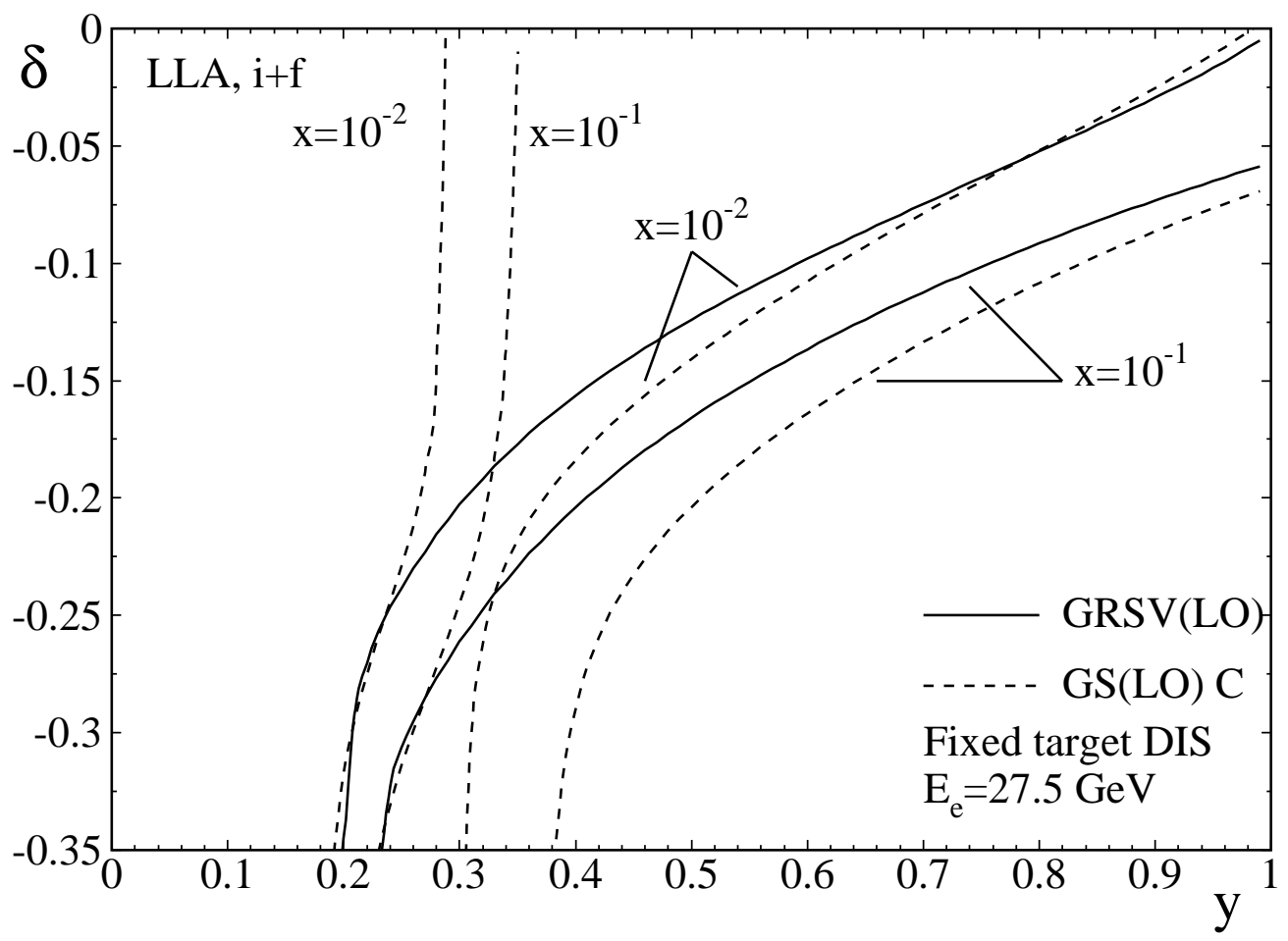

Figure 1: Radiative corrections to longitudinally polarized heavy quark electroproduction in $\mathcal{O}(\alpha)$-LLA at $\sqrt{S}=7.4 \mathrm{GeV}$ (HERMES). 


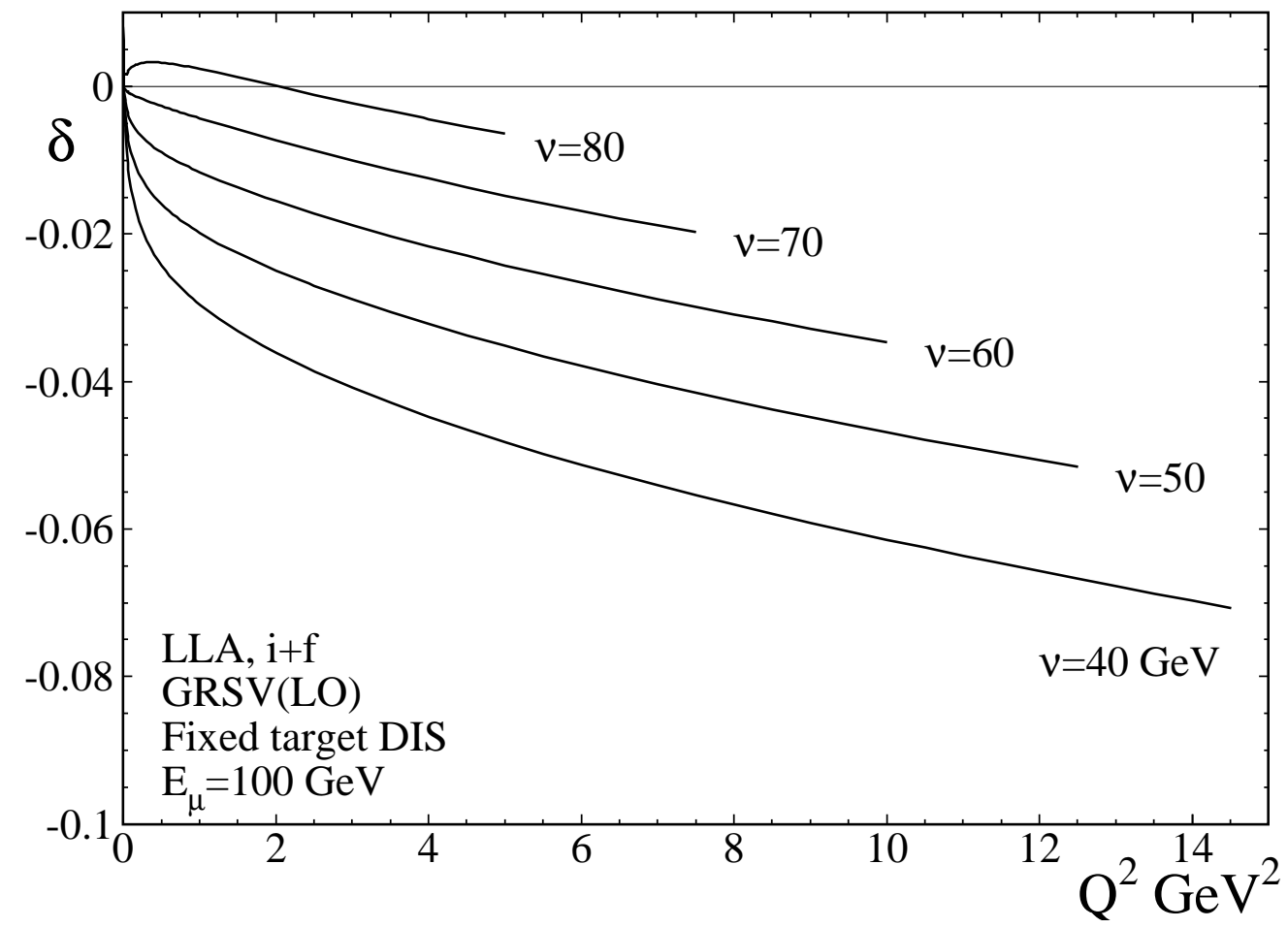

Figure 2: Radiative corrections to longitudinally polarized heavy quark muoproduction in $\mathcal{O}(\alpha)$-LLA at $\sqrt{S}=14.1 \mathrm{GeV}$ (COMPASS). 

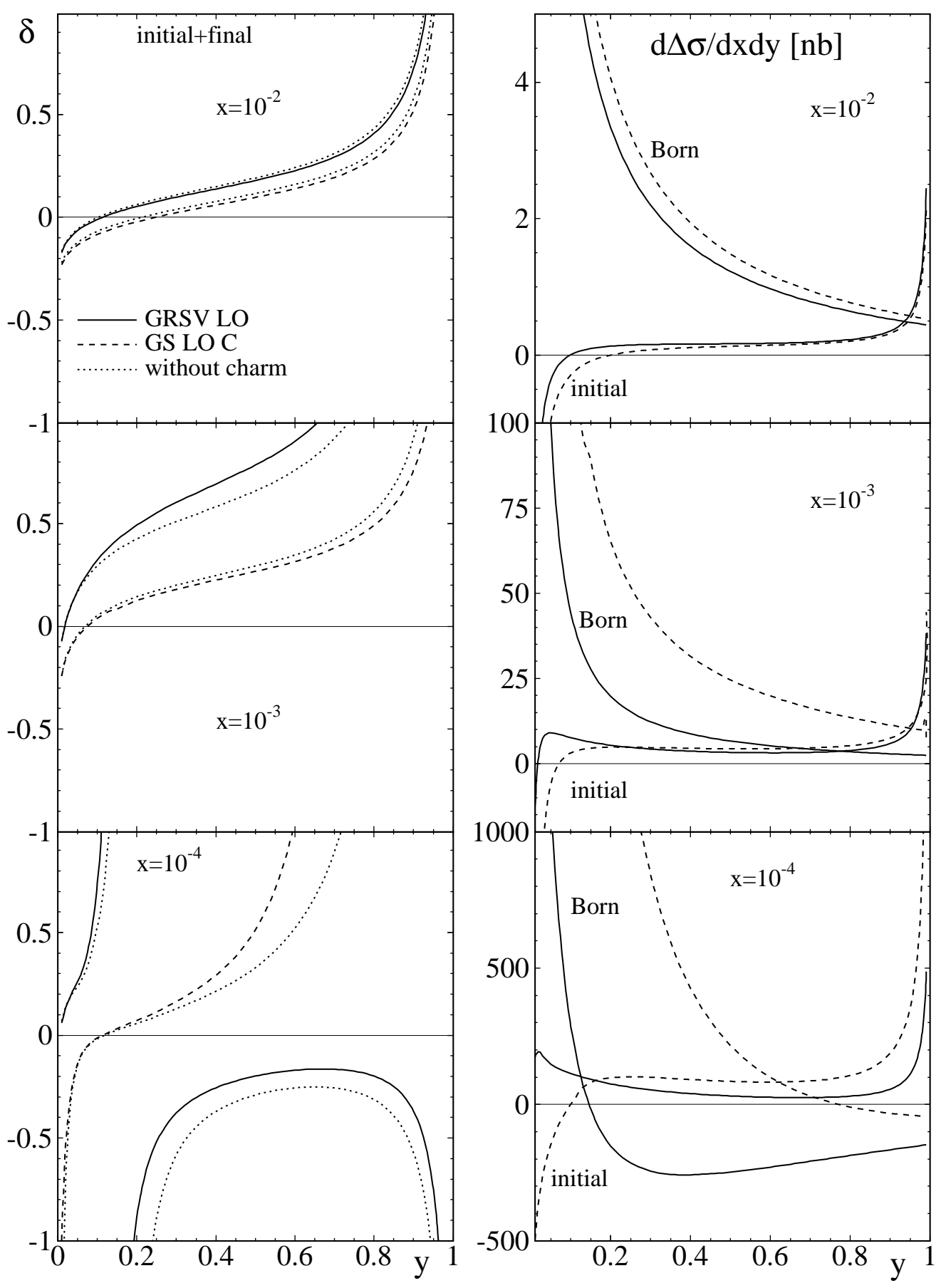

Figure 3: Right side: $d \Delta \sigma^{0, i}(e p \rightarrow e X)$ (u,d,s and heavy (charm) quark contribution) at $\sqrt{S}=300 \mathrm{GeV}$ for $x=10^{-2}, 10^{-3}$, and $10^{-4}$, using the GRSV(LO) 'S' [16] (solid line) and the GS(LO) 'C' [17](dashed line) parton densities and a factorization scale $\mu^{2}=Q^{2}+4 m_{c}^{2}$ with $m_{c}=1.5 \mathrm{GeV}$. Left side: The corresponding correction factor $\delta(x, y)$. The dotted lines have been calculated without heavy (charm) quark contribution. 

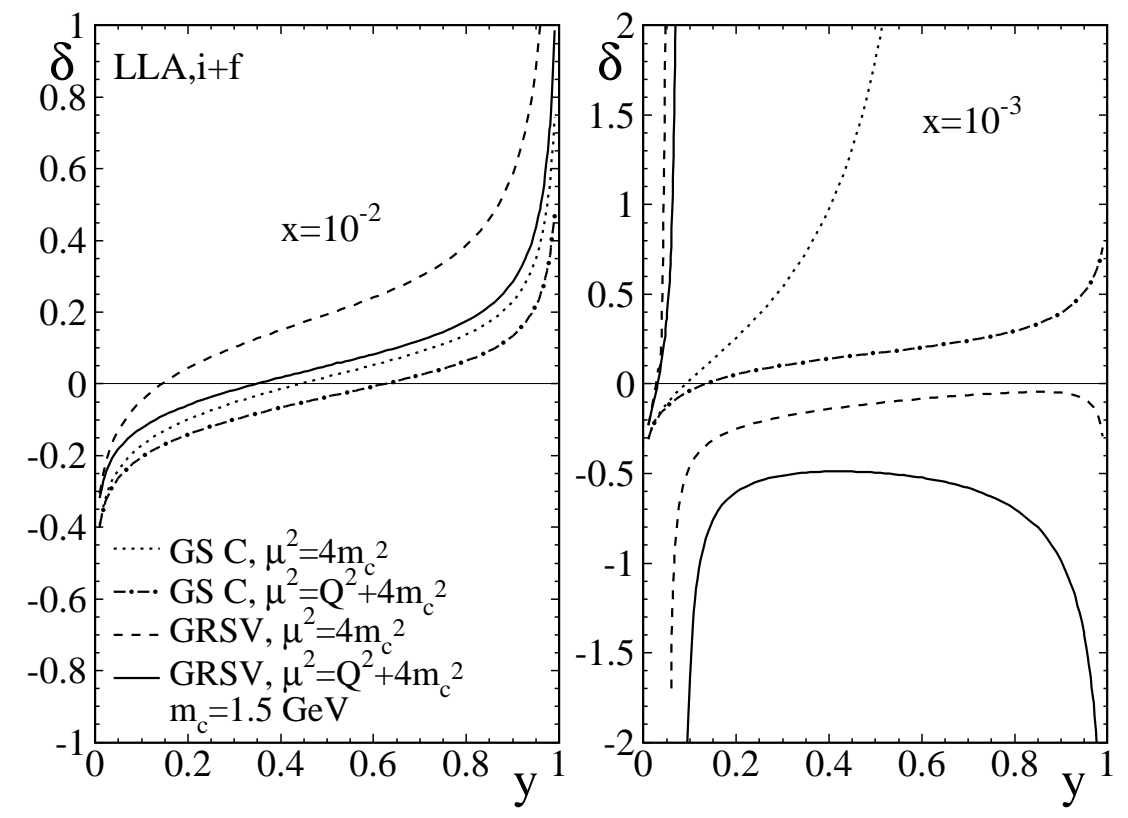

Figure 4: Radiative corrections to longitudinally polarized heavy quark electroproduction in $\mathcal{O}(\alpha)$-LLA at $\sqrt{S}=300 \mathrm{GeV}($ HERA).
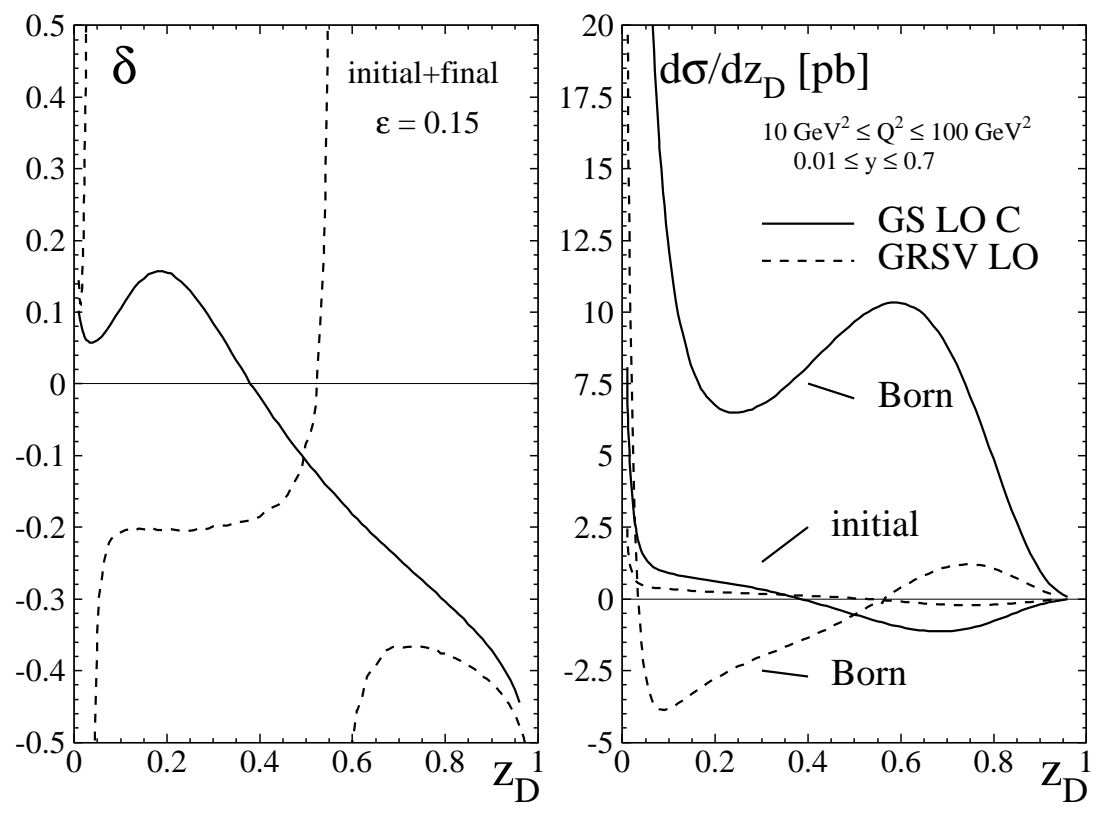

Figure 5: $z_{D}$-differential radiative corrections to deep inelastic charm production $\left(m_{c}=\right.$ $\left.1.5 \mathrm{GeV}, \mu^{2}=Q^{2}+4 m_{c}^{2}\right)$, integrated over the kinematical range $10 \mathrm{GeV}^{2} \leq Q^{2} \leq$ $100 \mathrm{GeV}^{2}, 0.01 \leq y \leq 0.7$, using the parton distributions GRSV(LO) 'S' [16] (solid line) and GS(LO) C [17] (dashed line) and a Peterson et al. fragmentation function [15] with $\varepsilon=0.15$. 